\title{
PREFACE
}

\section{Complementary and Alternative Approaches to Treating Communication Disorders}

\begin{abstract}
The National Center for Complementary and Alternative Medicine describes complementary and alternative medicine (CAM) as a group of diverse medical and health-care systems, practices, and products not presently considered to be part of conventional medicine. Although scientific evidence exists regarding some CAM therapies, for most, there are crucial questions yet to be answered. ${ }^{1}$ Medical disciplines are now addressing these key questions and CAM is becoming more and more accepted as a mainstream practice in several medical fields.
\end{abstract}

Last spring, I attended a conference for stroke survivors sponsored by the Rehabilitation and Recovery Committee of the Northeast Region of the American Stroke Association. The morning session was devoted to complementary and alternative methods of treating stroke. There were over 200 attendees, all very interested in hearing about the most recent advances in treating stroke using nontraditional methods. When the chair of the committee asked the audience members whether anyone in attendance had received any type of complementary/alternative treatment following their stroke, almost half of the survivors raised their hands. This response was consistent with a 1998 article from The New York Times reporting that an estimated $42 \%$ of American adults have tried some form of CAM. ${ }^{2}$ All of the survivors at the conference who had received CAM treatments (e.g., reiki, tai chi, massage, acupuncture, and electromagnetic therapy) received these as complementary or alternative approaches for treating their motor deficits in either physical therapy or occupational therapy. No survivors in the audience, however, had been offered a complementary or alternative treatment approach to their traditional speech-language treatment.

The field of CAM is a fast-growing component of many medical and rehabilitation programs. Medical schools across the country have incorporated CAM into their curricula; physical and occupational therapists now receive training in various CAM techniques. The National Institute for Health's funding for CAM research, through the National Center for Complementary and Alternative Medicine, is estimated to reach $\$ 116.2$ million in 2004. Yet, training in CAM for speechlanguage pathologists is scant. This issue of Seminars in Speech and Language is intended to offer some ways in which nontraditional therapies may be incorporated into traditional speech and language practices. In this issue, six articles describe new or nontraditional methods of treating and improving the overall wellbeing of individuals with communication disorders. The first article, written by me, provides an overview of nature-based therapy and its application to communication disorders. Three components of nature-based practice are addressed: animal-assisted therapy, horticultural therapy, and natural environment therapy.

Next, Murray and Kim review two forms of CAM: relaxation therapy and acupuncture. They present a thorough review of the literature on two nontraditional treatment approaches and discuss areas in need of further investigation.

The third article considers the role of exercise and volunteerism as a context for language and memory stimulation in individuals with Alzheimer's disease. The authors (Mahendra and Arkin) discuss the importance of a

Complementary and Alternative Approaches to Treating Communication Disorders; Editors in Chief, Nancy HelmEstabrooks, Sc.D., and Nan Bernstein Ratner, Ed.D.; Guest Editor, Kristine Lundgren, Sc.D. Seminars in Speech and Language, volume 25, number 2, 2004. ${ }^{1}$ Department of Neurology, Boston University School of Medicine, Harold Goodglass Aphasia Research Center, Boston, Massachusetts. Copyright (C) 2004 by Thieme Medical Publishers, Inc., 333 Seventh Avenue, New York, NY 10001, USA. Tel: +1(212) 584-4662. 0734-0478,p;2004,25,02,119,120,ftx,en;ss100191b. 
treatment approach that fosters improved and enhanced quality of life for individuals with Alzheimer's.

In the fourth article, Ramig, Fox, and Sapir address communication disorders associated with Parkinson's disease. They present preliminary data on an intensive behavioral treatment approach to target the underlying voice disorder in Parkinson's.

Martin and her colleagues describe the use of magnetic fields to create electrical currents that may suppress "over-activation" in discrete cortical regions. This technique (transcranial magnetic stimulation or TMS) is being used to treat a variety of disorders, but in this article Martin and associates concentrate on its application to treatment of communication deficits in stroke patients with nonfluent aphasia. They present preliminary findings from their current study of TMS.

In the final article, Klein and Albert review the neurochemistry of language and the neuropharmacology of aphasia, especially with regard to the effectiveness of drug treatments for aphasia. One important part of their discussion is the issue of which neurotransmitters work for which language functions in which patients, and why.

I hope that this issue of Seminars will increase the interest of clinicians and researchers in the use of nontraditional approaches for the treatment of communication disorders. Perhaps it will encourage readers to explore ways in which nontraditional approaches may be incorporated into speech-language pathology practices.

\section{REFERENCES}

1. National Center for Complementary and Alternative Medicine. What is Complementary and Alternative Medicine? Publication No. DC156. Bethesda, MD: National Center for Complementary and Alternative Medicine; 2002

2. Brody JE. Alternative medicine makes inroads, but watch out for the curves. New York Times. April 28, 1998:B9

Kristine Lundgren, Sc.D. Co-editor in Chief ${ }^{1}$ 